\section{Anti-prions and other agents}

SIR - Having observed an open reading frame in the non-coding strand of the PrP gene, Goldgaber in Scientific Correspondence ${ }^{1}$ predicts the presence of an anti-PrP protein and suggests the use of single-stranded RNA probes to detect expression of this anti-PrP gene.

We have carried out in situ hybridization experiments, using ${ }^{35} \mathrm{~S}$ labelled single-stranded RNA probes for exon 2 (protein-coding exon) and the large intron of the PrP gene ${ }^{2}$, to examine the expression of the PrP gene in the brain of scrapie-infected and uninfected mice and mouse embryos (our unpublished data). The presence of transcripts from the anti-PrP gene could not be detected by this method (see figure); if such transcripts are present they are at least

a
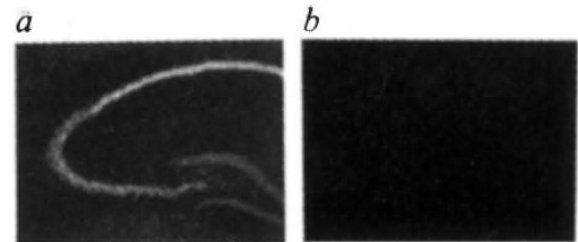

In situ hybridization of coronal sections through the brain of VM/DK (Sinc p7p7) mice, using ${ }^{35}$ S-labelled single-stranded RNA probes for exon 2 of PrP. $a$, Antisense and $b$, sense RNA probe. Hybridization was as visualized using nuclear track emulsion and dark-field microscopy.

100-fold less abundant than those of PrP. Further search for these transcripts will therefore require the use of more sensitive techniques involving strand-specific RNA amplification. Until then it may be premature to anticipate the anti-prion protein.

J. MANSON

J. HOPE

Institute for Animal Health,

AFRC and MRC Neuropathogenesis

Unit,

Ogston Building,

West Mains Road,

Edinburgh EH9 3JF, UK

1. Goldgaber, D. Nature 351, 106 (1991).

2. Westaway, D. et al. Cell 51, 651-662 (1987).

SIR - Goldgaber reported in Scientific Correspondence $^{1}$ that there is a large open reading frame on the opposite strand of the gene encoding the prion protein $(\mathrm{PrP})$. We had also made this observation and, because of our interest in bovine spongiform encephalopathy, we confirmed the presence of the putative anti-PrP gene in cattle by DNA nucleotide sequencing. To ascertain the expression of this gene, we hybridized single-stranded RNA probes specific to PrP and anti-PrP RNA with total cellular RNA extracted from various tissues

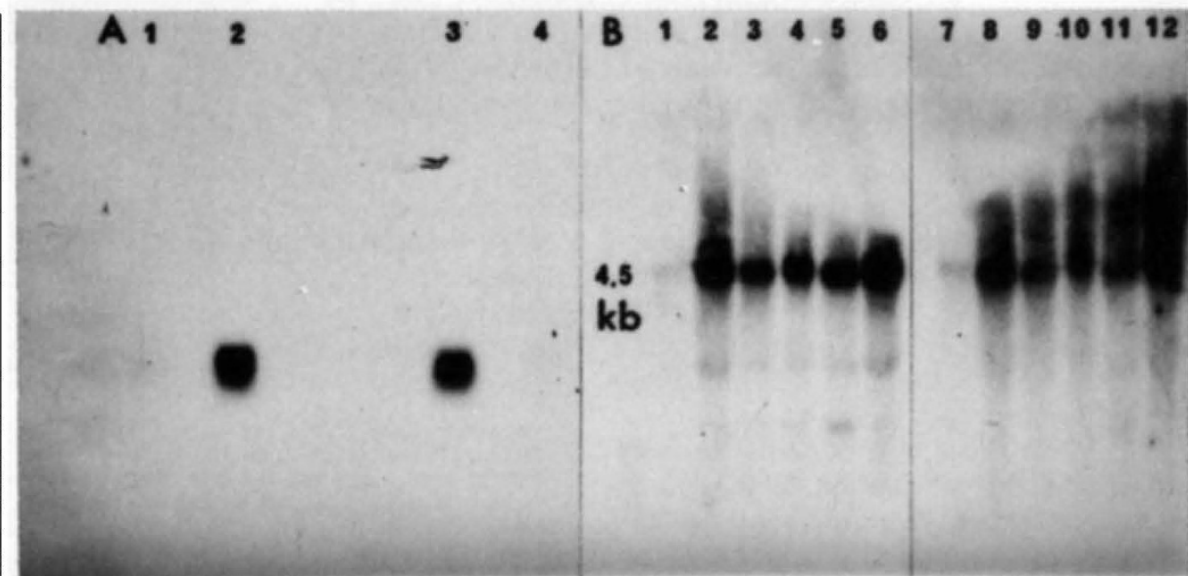

A, Probe specificity; Riboprobes were transcribed from the normal bovine PrP gene under the control of the T3 promoter of Bluescript SK (PrP strand specific) and KS (antisense strand specific). Templates were linearized to prevent erroneous transcription from the T7 promoter. Specificity was ensured by northern blotting unlabelled transcript derived from SK (tracks 1,3$)$ and KS $(2,4)$ strands. Tracks 1 and 2 were hybridized to a radiolabelled SK probe; tracks 3 and 4 were probed with KS. Densitometry of autoradiographs showed no cross hybridization. B, Analysis of total cellular RNA; northern-blotted filter contained bovine RNA from brain $(1,7)$, liver $(2,8)$, lung $(3,9)$, mesenteric lymph node $(4,10)$, heart muscle $(5,11)$ and spleen $(6,12)$. Tracks $1-6$ were hybridized to SK and $7-12$ probed with KS. Exposures were $1 \mathrm{~h}(1-6)$ and $12 \mathrm{~h}(7-12)$, respectively. Loadings of RNA were equivalent with the exception of brain which was approximately $1 / 10$ loading. Washing was to a stringency of $0.1 \times \mathrm{SSC}$ at $65^{\circ} \mathrm{C}$.

of a three-week-old calf. All tissues tested were positive with both probes (see figure), providing evidence for the presence of an anti-PrP RNA.

The presence of two mammalian genes transcribed from opposite strands at the same locus is not unique to the PrP gene. Adelman et al. reported ${ }^{2}$ that the rate gene encoding gonadotropinreleasing hormone is expressed in the central nervous system whereas a second gene, SH, is transcribed from the opposite strand in heart tissue. We note that in the genes for both PrP and gonadotrophic releasing hormone, the first base of the codon on one strand of DNA corresponds to the third base of the codon on the other strand. This arrangement provides a mechanism for maximum conservation of two proteins and might explain the high degree of interspecies conservation of the $\mathrm{PrP}$ gene.

Goldgaber suggests that the expression of the anti-PrP gene introduces "a new player" and presents a different view of spongiform encephalopathies. The hypothesis that two peptides encoded by complementary RNAs would assume conformations that resulted in specific and high-affinity binding of the pair has been postulated for several years $^{3-5}$. Consistent with this hypothesis is the fact that the hydrophobicity plot for anti-PrP is almost a mirror image of

1. Goldgaber, D. Nature 351, 106 (1991).

2. Adelman, J.P. et al. Science 235, 1514-1517 (1987).

3. Bost. K.L. et al. Proc. natn. Acad. Sci. U.S.A. 82, 1372-1375 (1985).

4. Blalock, J. E. \& Bost, K L. in: Molecular Neuroscience Expression of Neural Genes (eds Wong. F. et al.) 89-97 (Liss, New York. 1987).

5. Zull, E. Z. \& Smith, S. K. Trends biochem. Sci. 15, 257-261 (1990). that for $\operatorname{PrP}$. If anti-PrP protein is synthesized, it is possible that the two proteins do interact and that disruption of this interaction contributes to the pathogenesis of the transmissible spongiform encephalopathies.

R. G. HEWINSON

J. P. LOWINGS

M. D. DAWSON

M. J. WOODWARD

Central Veterinary Laboratory,

New Haw, Weybridge,

Surrey KT15 3NB, UK

SIR - New experimental evidence, summarized in News and Views by Weissmann $^{1}$, makes the protein-only model of the scrapie agent increasingly compelling. Although Weissmann provides a useful summary of some recent data, we would like to clarify the history of the protein-only model for the scrapie agent. Griffith in 1967 proposed $^{2}$ a detailed theoretical model describing a mechanism for the conversion of a normal host protein to a pathogenic form in scrapie. His proposal followed the suggestions by Alper and colleagues ${ }^{3}$ in 1967 that the scrapie agent may "...replicate without a nucleic acid", and by Pattison and Jones ${ }^{4}$ in the same year that the "scrapie transmissible agent may be, or may be associated with, a small basic protein". On the basis of his earlier work with natural scrapie in sheep, Parry had hypothesized that this disease could occur on a purely genetic basis and be secondarily transmissible ${ }^{5}$.

In 1982 Prusiner introduced the term 'prion' and outlined three mechanisms by which a foreign protein-only agent might replicate ${ }^{6}$; abnormal modification 